See discussions, stats, and author profiles for this publication at: https://www.researchgate.net/publication/335585543

\title{
Are Prisoners More Psychopathic than Non-forensic Populations? Profiling Psychopathic Traits among Prisoners, Community Adults, University Students, and Adolescents
}

Article in Deviant Behavior · January 2021

DOI: 10.1080/01639625.2019.1665221

CITATIONS

8

8 authors, including:

Daniel Boduszek

University of Huddersfield

131 PUBLICATIONS 1,754 CITATIONS

SEE PROFILE

Nicole Sherretts

University of Huddersfield

19 PUBliCATIONS 145 CITATIONS

SEE PROFILE
1,703

Agata Debowska

The University of Sheffield

54 PUBLICATIONS 693 CITATIONS

SEE PROFILE

Dominic Willmott

Manchester Metropolitan University

43 PUBLICATIONS 358 CITATIONS

SEE PROFILE

Some of the authors of this publication are also working on these related projects:

Project $\quad$ NoneinThree Project View project

Project Towards a Postmodern Concept of Spirituality within European Population View project 


\section{Are Prisoners More Psychopathic than Non- forensic Populations? Profiling Psychopathic Traits among Prisoners, Community Adults, University Students, and Adolescents}

Daniel Boduszek, Agata Debowska, Nicole Sherretts, Dominic Willmott, Mike Boulton , Krzysztof Kielkiewicz , Katarzyna Popiolek \& Philip Hyland

To cite this article: Daniel Boduszek, Agata Debowska, Nicole Sherretts , Dominic Willmott , Mike Boulton , Krzysztof Kielkiewicz , Katarzyna Popiolek \& Philip Hyland (2021) Are Prisoners More Psychopathic than Non-forensic Populations? Profiling Psychopathic Traits among Prisoners, Community Adults, University Students, and Adolescents, Deviant Behavior, 42:2, 232-244, DOI: 10.1080/01639625.2019.1665221

To link to this article: https://doi.org/10.1080/01639625.2019.1665221

Published online: 12 Sep 2019.

Џll Article views: 366

View Crossmark data [־

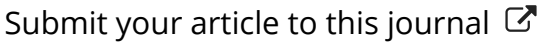

Q View related articles $\sqsubset$

Citing articles: 2 View citing articles 지 


\title{
Are Prisoners More Psychopathic than Non-forensic Populations? Profiling Psychopathic Traits among Prisoners, Community Adults, University Students, and Adolescents
}

\author{
Daniel Boduszek (1) a,b , Agata Debowskac, Nicole Sherretts ${ }^{a}$, Dominic Willmott ${ }^{\mathrm{a}}$, Mike Boulton ${ }^{\mathrm{d}}$, \\ Krzysztof Kielkiewicze ${ }^{\mathrm{e}}$ Katarzyna Popiolek ${ }^{\mathrm{b}}$, and Philip Hyland ${ }^{\mathrm{f}}$ \\ aUniversity of Huddersfield, Huddersfield, UK; bSWPS University of Social Sciences and Humanities, Katowice, Poland; \\ 'University of Sheffield, Sheffield, UK; dUniversity of Chester, Chester, UK; eUniversity of Finance and Management, \\ Warsaw, Poland; fMaynooth University, Dublin, Ireland
}

\begin{abstract}
The aim of this study was to compare prisoners $(n=772)$, community adults ( $n=1201)$, university students $(n=2080)$, and adolescents $(n=472)$ on four sets of psychopathic traits (affective responsiveness, cognitive responsiveness, interpersonal manipulation, and egocentricity), using a psychopathy measure which does not index criminal/antisocial behavior - the Psychopathic Personality Traits Scale. Another aim was to examine patterns of co-occurrence between psychopathic personality traits among offending and non-offending populations. ANOVA results indicated significant differences between the four groups on all PPTS dimensions. Adolescents demonstrated greater deficits in affective and cognitive responsiveness than the remaining groups of participants. Prisoners had elevated deficits in cognitive responsiveness, compared with university students and community adults. University students scored higher on interpersonal manipulation than adolescents and prisoners, and higher on egocentricity than community adults and prisoners. Latent profile analysis revealed four distinct classes of psychopathic traits among all samples, although not all classes were qualitatively equivalent across samples. Low psychopathy groups were identified for all samples. There were clear high psychopathy groups for prisoners and university students, with approximately $7 \%$ of prisoners and students belonging in the groups. This finding indicates that past research could have over-estimated the prevalence of psychopathy in forensic populations due to inclusion of criminal behavior items in psychopathy assessment.
\end{abstract}

\section{ARTICLE HISTORY}

Received 30 April 2019

Accepted 4 August 2019

\section{Introduction}

Psychopathy, although not included in the most recent, fifth edition of the Diagnostic and statistical manual of mental disorders (DSM-5; American Psychiatric Association [APA] 2013), is referred to as one of the oldest mental disorders (Buzina 2012). The concept of psychopathy has aroused increasing interest in researchers and practitioners, however, there is lack of agreement on what constitutes the disorder and how it should be assessed (Ogloff 2006). More specifically, as long as there appears to be a consensus among researchers that psychopaths are characterized by callous affect, lack of empathy, and manipulativeness, the inclusion of criminal/antisocial behavior as a fundamental component of the psychopathy construct remains contentious (see Boduszek and Debowska 2016 for a critical review).

CONTACT Daniel Boduszek d.boduszek@hud.ac.uk @ Department of Psychology, University of Huddersfield, Edith Key Building, Queensgate, Huddersfield HD1 3DH, UK

Color versions of one or more of the figures in the article can be found online at www.tandfonline.com/udbh. 
To date, the most widely used psychopathy assessment tool has been the Psychopathy Checklist (PCL; Hare 1980) and its more recent version - the Psychopathy Checklist - Revised (PCL-R; Hare 1991, 2003). The measure reflects a psychopathy construct composed of four sets of traits: interpersonal manipulation (e.g., deceitfulness, superficial charm, grandiosity), callous affect (e.g., lack of empathy, remorse, or guilt), erratic lifestyle (e.g., impulsivity, irresponsibility), and antisocial behavior (e.g., social deviance, criminality). The PCL-R, consisting of 20 items, is administered and completed by a trained clinician. All items are indexed on a three-point scale, with scores varying from 0 to 40. A cutoff score of 30 has been suggested for diagnosing psychopathy (Hare and Neumann 2008). The PCL-R-based estimated prevalence of psychopathy in the general population is between $0.3-2 \%$, with slightly higher rates for males $1-2 \%$ than females $0.3-0.7 \%$ (Patrick and Drislane 2015). The prevalence of psychopathy among general offender population, on the other hand, has been reported to oscillate between 15-25\% (Lilienfeld and Arkowitz 2007; Woodworth and Porter 2002). In considering the inclusion of lifestyle and antisocial items in the PCL-R as well as the fact that psychopathy diagnosis is based on a score calculated for the full scale, the discrepancy between general and offending populations in the occurrence of the disorder is unsurprising. It is now increasingly recognized that using the PCL-R for psychopathy assessment, could have resulted in an overestimation of the disorder in samples who offend (Boduszek and Debowska 2016; Edens et al. 2001; Patrick 2007; Patrick et al. 2007; Rogers 1995).

Several sources have indicated that antisocial/criminal behavior should be regarded as a possible consequence rather than an integral part of psychopathy (e.g., Boduszek and Debowska 2016; Boduszek, Debowska, and Willmott 2017; Boduszek et al., 2016a; Cooke and Michie 2001; Debowska et al. 2018; Sharratt, Boduszek, and Retzler 2019; Skeem and Cooke 2010a, 2010b). In line with this argument, Boduszek et al. (2016a) proposed a non-behavioral assessment of psychopathy - the Psychopathic Personality Traits Scale (PPTS). The PPTS is a 20-item self-report instrument composed of four dimensions: affective responsiveness (i.e., deficits in emotional reactions to others), cognitive responsiveness (i.e., deficits in cognitive engagement with others), interpersonal manipulation (i.e., deceptive and coercive communicative style), and egocentricity (i.e., focusing on own interests and beliefs). The exclusion of behavioral aspects renders the scale suitable for use with a variety of samples, including non-offending populations and young people. Although children and adolescents cannot be diagnosed with a personality disorder, recognizing certain problems early on and gaining a deeper understanding of trait stability across developmental stages, could be advantageous to designing prompt and efficient intervention strategies (Frick 2007). In considering the multi-dimensional nature of the PPTS and the assertion that psychopathy measures, especially those used among young people, should be of a continuous rather than categorical character (Marcus 2017), a total cutoff score for psychopathy has not been introduced. Instead, qualitative and quantitative differences in the expression of psychopathic traits can be explored with the use of person-centered analytic techniques, such as latent profile analysis (LPA). LPA has the power to reveal variations in trait intensity as well as grouping of traits across individuals. Unlike traditional variable-centered statistical approaches, LPA does not assume independence among observed variables (De Fruyt and De Clercq. 2014; Shevlin and Elklit 2008). In LPA environment, associations among a set of indicators are said to be explained by a finite number of mutually exclusive groups/classes. The optimal number of classes is selected based on multiple model assessments (Nylund, Asparouhov, and Muthén 2007).

To date, only one study inquired into latent classes of psychopathy using the PPTS. Specifically, Boduszek, Debowska, and Willmott (2017) aimed to detect meaningful subtypes of psychopathy in a large, systematically selected sample of adult male offenders incarcerated in 5 medium and 5 maximum security prisons in Poland $(N=1126)$. The results yielded a 5 -class solution, with varying levels and combinations of psychopathic personality traits across classes. Class 1 was characterized by low mean scores on all four PPTS dimensions and was termed the "low psychopathy group". The group consisted of $44.6 \%$ of prisoners and was the largest group in the study. Class 2 (16.8\% of prisoners), labeled the "moderate affective/cognitive responsiveness group", was characterized by 
moderate mean scores on affective and cognitive responsiveness and relatively low ratings on interpersonal manipulation and egocentricity. Class 3 (20.8\% of prisoners), labeled the "high interpersonal manipulation group", was characterized by low mean scores on affective responsiveness, cognitive responsiveness, and egocentricity and high on interpersonal manipulation. Class 4 (10.8\% of prisoners), labeled the "moderate psychopathy group", was characterized by moderate mean scores on affective responsiveness, cognitive responsiveness, and egocentricity and high interpersonal manipulation. Class 5 was the "high psychopathy group", characterized by very high mean scores on affective responsiveness, moderate cognitive responsiveness, and high interpersonal manipulation and egocentricity. Consisting of $7.1 \%$ of inmates, this was the smallest group in the analysis. This result indicates that $(a)$ most prisoners, even those from medium and maximum security prisons, are not psychopathic and $(b)$ psychopathy could have been over-diagnosed in forensic populations due to indexing criminal behavior in psychopathy assessment. This important finding, however, remains to be verified with more diverse offending samples. No prior research with the PPTS focused on profiling psychopathic personality traits among non-offending populations, presenting another void in the literature.

\section{Study aims}

It appears that the much higher prevalence of psychopathy among forensic than non-forensic populations can be a function of the inclusion of criminal/antisocial behavior items in psychopathy measurement (Boduszek and Debowska 2016). Additionally, the use of behaviorally-based and categorical measures, especially among young people, remains controversial, due to the stigma attached to psychopathy diagnosis, particularly in relation to risk of violence (Marcus 2017). Therefore, in the present study, we examined psychopathy using a personality-based measure without a cutoff point for psychopathy diagnosis, the PPTS (Boduszek et al. 2016a). The first aim was to compare prisoners, community adults, university students, and adolescents on four PPTS dimensions (affective responsiveness, cognitive responsiveness, interpersonal manipulation, and egocentricity). Another aim was to expand the current understanding of intensity and permutations of psychopathic personality traits among offending and non-offending samples, without diagnostic labeling. To expand on Boduszek, Debowska, and Willmott's (2017) LPA study with Polish inmates whose results indicated that approximately 7\% of prisoners can be classed as highly psychopathic, we conducted a similar analysis in a sample of U.S. prisoners. Next, to establish whether qualitatively diverse constellations of psychopathic traits exist across populations, we performed LPA using samples of community adults, university students, and adolescents. Given the paucity of research in the area, we made no specific predictions as to the number of latent classes. We did, however, predict that a low and high psychopathy group would be found for all samples recruited and that these groups would be the most and least numerous of all classes respectively.

\section{Method}

\section{Sample and procedure}

In this study, we applied opportunity sampling procedure using self-report survey design. The data was collected from four independent groups of participants on different occasions between year 2015-2017. Ethical approval for the study was granted by relevant institutional panels (Sample 1 = Pennsylvania Department of Corrections Research Review Committee, Sample 2, 3 and 4 = School Research Ethics Panel at University of Huddersfield \& Psychology Department Research Ethics Panel at University of Chester). Participation was voluntary and anonymous and written informed consent was obtained from all individuals involved in the study and from the parents/legal guardians of all non-adult participants. Respondents were informed that they could withdraw from the study at any time until questionnaire submission and that providing specific 
reasons for not participating/withdrawing was not required. All participants were debriefed at the end of the study. The total sample size consisted of 4525 participants.

Sample 1 consisted of 772 offenders incarcerated in two maximum and one medium security prisons in Pennsylvania (USA). The sample included 434 males and 338 females. The age range was between 20 and 77 years $(M=38.82, S D=10.95, M d n=37$, and Mode $=34)$. Printed self-report surveys were distributed among inmates by prison personnel. Respondents were instructed to place completed surveys in envelopes and return them to a data collector.

Sample 2 consisted of 1201 community adults from North England (UK), including 384 males and 817 females. Participants ranged in age from 21 to $70(M=32.18, S D=10.69, M d n=26$, and Mode $=25$ ). Participants completed the survey online.

Sample 3 consisted of 2080 university students from two universities from North England, including 548 males and 1532 females (mature students were not included in the analysis). Students ranged in age from 18 to $23(M=20.33, S D=1.38, M d n=20$, and Mode $=20)$. Participants completed the survey online.

Sample 4 consisted of 472 adolescents from one secondary school located in North England. The sample included 257 boys and 215 girls. Participants ranged in age from 12 to 15 years $(M=13.05$, $S D=0.84, M d n=13$, and Mode = 14). Prior to participation, parents' informed consent was obtained as well as children assent. Participants completed printed surveys in the school setting. Completed surveys were collected by a researcher.

\section{Measures}

\section{Psychopathic personality traits scale}

(PPTS; Boduszek et al. 2016a) is a personality-based self-reported 20-item measure designed to assess psychopathic traits in forensic and non-forensic populations. The scale was developed to measure four factors labeled affective responsiveness, cognitive responsiveness, interpersonal manipulation, and egocentricity. Each subscale consists of five items measured using "agree" (1) and "disagree" (0) format (i.e., a trait is either present or absent). Total scale scores range from 0 to 20 , whereas subscale scores range from 0 to 5 . Higher scores indicate increased levels of psychopathic personality traits (i.e., increased egocentricity and interpersonal manipulation and increased deficits in affective and cognitive responsiveness). Sample scale items include: “I don't care if I upset someone to get what I want" (affective responsiveness); "Before criticizing somebody, I try to imagine and understand how it would make them feel" (cognitive responsiveness); "I know how to pay someone compliments to get something out of them" (interpersonal manipulation); "In general, I'm only willing to help other people if doing so will benefit me as well" (egocentricity). Six scale items are reverse-scored. Internal reliability of the PPTS factors was assessed using composite reliability. Results suggest that all four psychopathy factors (affective responsiveness $=0.78$, cognitive responsiveness $=0.86$, interpersonal manipulation $=0.83$, and egocentricity $=0.69$ ) demonstrate good internal reliability. The PPTS is an established and validated measure (see Boduszek et al. 2016a; Boduszek et al. 2018) (Boduszek, Debowska, and Willmott 2018).

\section{Analysis}

ANOVA was used to determine statistical differences between prisoners, community adults, university students, and adolescents on the four PPTS subscales. Effect size was calculated using Cohen's $d$. Cohen (1988) suggested that $d=0.2$ be considered a "small" effect size, 0.5 represents a "medium" effect size and 0.8 a "large" effect size.

Latent profile analysis (LPA) was used to identify homogeneous groups (latent classes) from four independent data sets using four dimensions of the PPTS. The aim of the LPA was to determine the number of psychopathy classes and verify whether they differed qualitatively and/or quantitatively. The LPA used four total psychopathy scores for each of the four psychopathy dimensions of the PPTS 
(affective responsiveness, cognitive responsiveness, interpersonal manipulation, and egocentricity). Five alternative models were assessed (a one-class model through to a five-class model) using robust maximum likelihood (Yuan and Bentler 2000). To avoid solutions based on local maxima, 500 random sets of starting values were used initially and 100 final stage optimizations. The relative fit of the models was compared using the Akaike Information Criterion (AIC; Akaike 1987), the Bayesian Information Criterion (BIC; Schwarz 1978), and sample size adjusted Bayesian Information Criterion (SSA-BIC; Sclove 1987). The model with the lowest value indicates the best latent profile solution. We also calculated entropy value which indicates the ability of the model to correctly classify participants, with higher values indicating better classification (Ramaswamy et al. 1993). In addition, we used the LoMendell-Rubin adjusted likelihood ratio test (LMR; Lo, Mendell, and Rubin 2001) to compare models with increasing numbers of latent classes. A non-significant value $(p>.05)$ suggests that the model with one less class should be accepted. All analyses were conducted on the full sample and four subgroups (prisoners, community adults, university students, and adolescents) using Mplus version 7.4.

\section{Results}

Descriptive statistics for four factors of psychopathy for all groups are presented in Table 1 . Additionally, ANOVA results showed statistically significant differences between groups on all psychopathy variables. Adolescents scored higher than university students $(d=1.02)$, community adults $(d=1.02)$ and prisoners $(d=0.95)$ on affective responsiveness, demonstrating greater deficits in this domain. In relation to cognitive responsiveness, adolescents scored higher than university students $(d=2.04)$, community adults $(d=2.09)$ and prisoners $(d=1.80)$, whereas prisoners scored higher than university students $(d=0.26)$ and community adults $(\mathrm{d}=0.31)$. University students scored higher than adolescents $(d=0.47)$ and prisoners $(d=0.40)$, whereas community adults scored higher than prisoners $(d=0.42)$ and adolescents $(d=0.49)$ on interpersonal manipulation. In terms of egocentricity, university students displayed higher scores than community adults $(d=.23)$ and prisoners $(d=.14)$.

The fit statistics for the latent profile analysis of psychopathy performed on the full sample are presented in Table 2. Based on AIC, BIC, and SSA-BIC values we were unable to identify the best model. However, the LMR shows that there is no significant improvement in fit for the five-class solution therefore, the four-class solution is preferred. The entropy test confirms the supremacy of the four-class solution over alternative solutions. Based on these statistics, the four-class solution is considered the best fitting model for the full sample. Additionally, latent profile analysis was conducted on four subsamples and all results confirmed the four-class solution: prison sample entropy $=0.84$, community sample - entropy $=0.91$, university sample - entropy $=0.91$, young people - entropy $=0.91$.

Figure 1 shows the profile plot for the four-class solution (means and standard deviations are presented in Table 3 ) for the prison sample. Class 1 (53.7\% of prisoners) is the largest group. It is

Table 1. Descriptive statistics and ANOVA results for prison sample, community sample, university sample, and adolescent sample.

\begin{tabular}{|c|c|c|c|c|c|c|}
\hline Variable & $\begin{array}{c}\text { Prison } \\
(P) \\
M(S D)\end{array}$ & $\begin{array}{c}\text { Community } \\
\text { (C) } \\
M(S D)\end{array}$ & $\begin{array}{c}\text { University } \\
(U) \\
M(S D)\end{array}$ & $\begin{array}{c}\text { Adolescent } \\
\text { (A) } \\
M(S D)\end{array}$ & $F$ ratio & Significant differences (Cohen's $d$ ) \\
\hline $\begin{array}{l}\text { Affective } \\
\quad \text { responsiveness }\end{array}$ & $\begin{array}{c}1.01 \\
(1.26)\end{array}$ & $1.02(1.11)$ & $.97(1.20)$ & $2.05(.89)$ & $115.15^{*}$ & $\mathrm{~A}>\mathrm{U}(1.02) ; \mathrm{A}>\mathrm{C}(1.02) ; \mathrm{A}>\mathrm{P}(0.95)$ \\
\hline $\begin{array}{l}\text { Cognitive } \\
\text { responsiveness }\end{array}$ & $\begin{array}{c}1.22 \\
(1.16)\end{array}$ & $.86(1.19)$ & $.92(1.18)$ & $3.29(1.14)$ & $554.96^{*}$ & $\begin{array}{c}\mathrm{A}>\mathrm{U}(2.04) ; \mathrm{A}>\mathrm{C}(2.09) ; \mathrm{A}>\mathrm{P}(1.80) ; P>\mathrm{U} \\
(0.26) ; P>C(0.31)\end{array}$ \\
\hline $\begin{array}{l}\text { Interpersonal } \\
\text { manipulation }\end{array}$ & $\begin{array}{l}1.66 \\
(1.57)\end{array}$ & $2.31(1.54)$ & $2.29(1.56)$ & $1.57(1.49)$ & $55.79^{*}$ & $\begin{array}{c}\mathrm{U}>\mathrm{A}(0.47) ; \mathrm{U}>\mathrm{P}(0.40) ; \mathrm{C}>\mathrm{P}(0.42) ; \mathrm{C}>\mathrm{A} \\
(0.49)\end{array}$ \\
\hline Egocentricity & $\begin{array}{c}1.93 \\
(1.22)\end{array}$ & $1.81(1.24)$ & $2.10(1.26)$ & $1.93(1.29)$ & $14.02^{*}$ & $U>C(0.23) ; U>P(0.14)$ \\
\hline
\end{tabular}

Note. Bonferroni correction * $p<.013 . d=0.2$ "small" effect size, 0.5 "medium" effect size, and 0.8 "large" effect size. 
Table 2. Fit indices for the latent profile analysis of the four psychopathy factors for the full sample $(\mathrm{N}=4525)$.

\begin{tabular}{lcccccc}
\hline Model & AIC & BIC & SSA-BIC & Entropy & LMR & $p$ \\
\hline 1 & 61168.97 & 61220.30 & 61194.88 & n/a & n/a & n/a \\
2 & 58674.36 & 58757.77 & 58716.47 & 0.860 & 2446.46 & $<0.001$ \\
3 & 57625.60 & 57741.09 & 57683.90 & 0.860 & 1034.18 & $<0.001$ \\
$\mathbf{4}$ & $\mathbf{5 7 0 3 4 . 1 5}$ & $\mathbf{5 7 1 8 1 . 7 2}$ & $\mathbf{5 7 1 0 8 . 6 4}$ & $\mathbf{0 . 9 0 4}$ & $\mathbf{5 8 7 . 4 8}$ & $\mathbf{0 . 0 2}$ \\
5 & 56408.99 & 56588.64 & 56499.67 & 0.875 & 203.209 & 0.16 \\
\hline
\end{tabular}

Note. AIC = Akaike information criterion; BIC = Bayesian information criterion; SSA-BIC = sample size adjusted BIC; LMR = LoMendell-Rubin's adjusted likelihood ratio test. Boldface type suggests the best-fitting model.

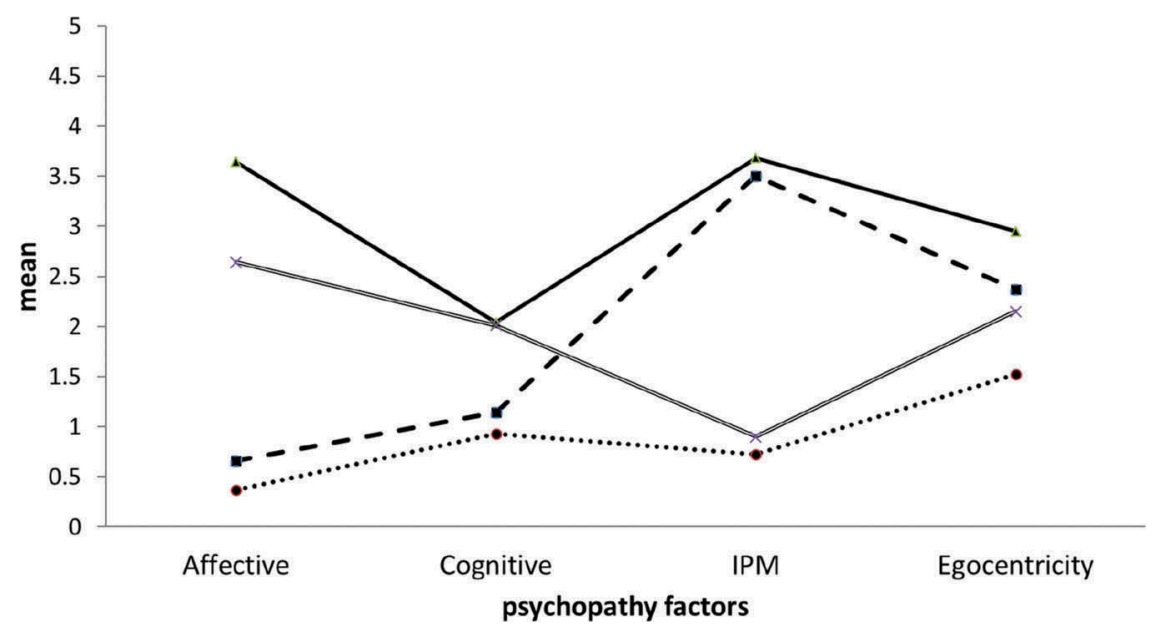

Figure 1. Latent profile analysis plot of psychopathic traits in prison sample $(N=772)$. Dotted line - Class $1=$ "low psychopathy group" (53.7\% of cases); Dashed line - Class 2 = "moderate egocentricity and high interpersonal manipulation group" (24.9\%); Double solid line - Class 3 = "moderate psychopathy group" (13.8\%); solid line - Class 4 = "high psychopathy group" (7.6\%); Affective $=$ affective responsiveness factor; Cognitive $=$ cognitive responsiveness; IPM $=$ interpersonal manipulation.

characterized by low mean scores on all four psychopathy dimensions and is labeled the "low psychopathy group". Class $2(24.9 \%)$ is characterized by low mean scores on affective and cognitive responsiveness, moderate on egocentricity and high on interpersonal manipulation. This class is labeled the "moderate egocentricity and high interpersonal manipulation group". Class 3 (13.8\%) is characterized by moderate mean scores on affective responsiveness, cognitive responsiveness, and egocentricity and low on interpersonal manipulation. This class is labeled the "moderate psychopathy group". Class $4(7.6 \%)$ is the smallest group. It is characterized by high mean scores on affective responsiveness, interpersonal manipulation, egocentricity moderate scores on cognitive responsiveness. This class is labeled the "high psychopathy group".

Figure 2 shows the profile plot for the four-class solution (means and standard deviations are presented in Table 3) for adult community sample. Class 1 ( $73 \%$ of adults) is the largest group. It is characterized by low mean scores on affective responsiveness, cognitive responsiveness, egocentricity, and moderate scores on interpersonal manipulation and, as such, is labeled the "low psychopathy group". Class $2(14.2 \%)$ is characterized by low mean scores on affective responsiveness and moderate scores on cognitive responsiveness, interpersonal manipulation and egocentricity. This class is labeled the "moderate psychopathy group". Class $3(6.9 \%)$ is characterized by high interpersonal manipulation and egocentricity scores, and moderate affective responsiveness and low cognitive responsiveness scores. This class is labeled the "high interpersonal manipulation and egocentricity psychopathy group". Class 4 (5.9\%) is the smallest group. It is characterized by high mean scores on cognitive responsiveness, and moderate scores on interpersonal manipulation, 
Table 3. Means (Standard Deviations) for the Four-Class Solution of the Psychopathic Personality Traits Scale (PPTS) in prison, community, university, and adolescent sample.

\begin{tabular}{|c|c|c|c|c|}
\hline Models & $\begin{array}{l}\text { AR } \\
M(S D)\end{array}$ & $\begin{array}{l}\mathrm{CR} \\
M(S D)\end{array}$ & $\begin{array}{l}\text { IPM } \\
M(S D)\end{array}$ & $\begin{array}{l}\mathrm{EGO} \\
M(S D)\end{array}$ \\
\hline \multicolumn{5}{|c|}{ Prison $(N=772)$} \\
\hline Class 1 & $0.66(0.67)$ & $1.14(1.07)$ & $3.50(0.86)$ & $2.37(1.13)$ \\
\hline Class 2 & $0.37(0.67)$ & $0.93(1.07)$ & $0.72(0.86)$ & $1.52(1.13)$ \\
\hline Class 3 & $3.64(0.67)$ & $2.04(1.07)$ & $3.68(0.86)$ & $2.95(1.13)$ \\
\hline Class 4 & $2.64(0.67)$ & $2.01(1.07)$ & $0.90(0.86)$ & $2.15(1.13)$ \\
\hline \multicolumn{5}{|c|}{ Community $(N=1201)$} \\
\hline Class 1 & $0.73(0.94)$ & $0.26(0.48)$ & $2.18(1.47)$ & $1.56(1.11)$ \\
\hline Class 2 & $2.53(0.94)$ & $1.45(0.48)$ & $3.83(1.47)$ & $3.46(1.11)$ \\
\hline Class 3 & $1.11(0.94)$ & $2.34(0.48)$ & $1.98(1.47)$ & $1.93(1.11)$ \\
\hline Class 4 & $2.51(0.94)$ & $4.00(0.48)$ & $2.86(1.47)$ & $2.78(1.11)$ \\
\hline \multicolumn{5}{|c|}{ University $(N=2080)$} \\
\hline Class 1 & $0.28(0.53)$ & $0.37(0.68)$ & $1.98(1.49)$ & 1.78 (1.16) \\
\hline Class 2 & $2.36(0.53)$ & $0.65(0.68)$ & $2.71(1.49)$ & $2.78(1.16)$ \\
\hline Class 3 & $3.69(0.53)$ & $2.54(0.68)$ & $3.60(1.49)$ & 3.37 (1.16) \\
\hline Class 4 & $1.17(0.53)$ & $2.91(0.68)$ & $2.55(1.49)$ & 2.51 (1.16) \\
\hline \multicolumn{5}{|c|}{ Adolescent $(N=472)$} \\
\hline Class 1 & $2.13(0.83)$ & $3.26(0.92)$ & $2.37(0.53)$ & $2.30(1.17)$ \\
\hline Class 2 & $0.61(0.83)$ & $0.54(0.92)$ & $0.52(0.53)$ & $0.97(1.17)$ \\
\hline Class 3 & $2.35(0.83)$ & $2.93(0.92)$ & $4.33(0.53)$ & $2.83(1.17)$ \\
\hline Class 4 & $2.05(0.83)$ & $3.67(0.92)$ & $0.36(0.53)$ & 1.52 (1.16) \\
\hline
\end{tabular}

Note. $\mathrm{AR}=$ Affective responsiveness, $\mathrm{CR}=$ Cognitive responsiveness, IPM = Interpersonal Manipulation, $\mathrm{EGO}=$ Egocentricity.

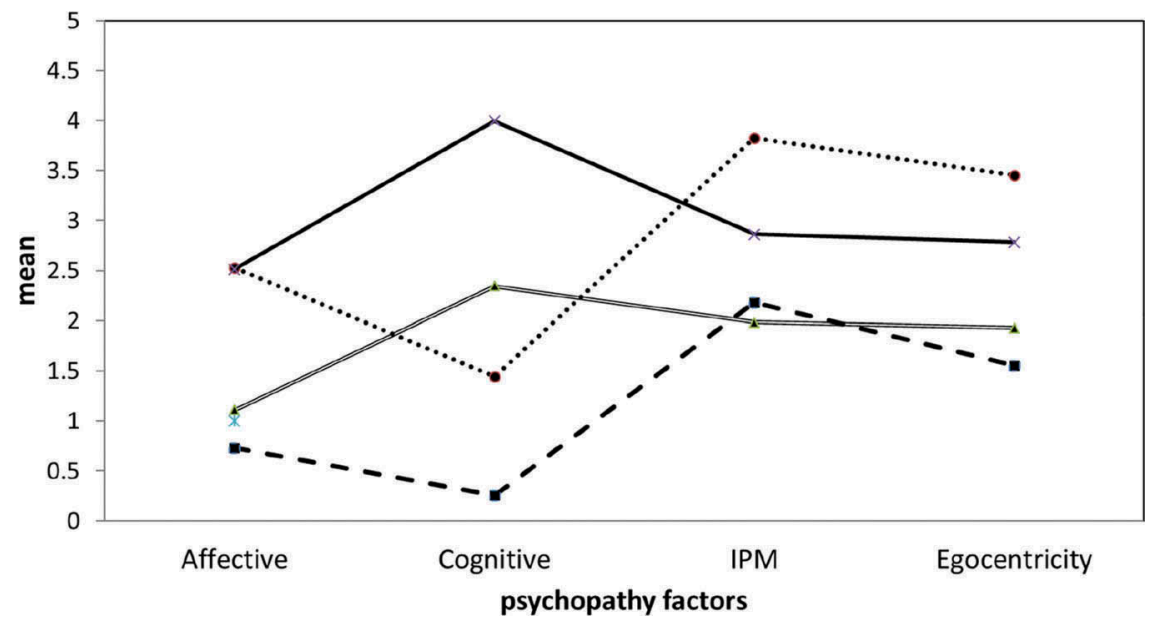

Figure 2. Latent profile analysis plot of psychopathic traits in community sample $(N=1201)$. Dashed line - Class $1=$ "low psychopathy group" (73\% of cases); Double solid line - Class 2 = "moderate psychopathy group" (14.2\%); Dotted line - Class 3 = "high interpersonal manipulation and egocentricity psychopathy group" (6.9\%); Solid line - Class $4=$ "high cognitive responsiveness and moderate other psychopathy factors group" (5.9\%); Affective = affective responsiveness factor; Cognitive $=$ cognitive responsiveness factor; IPM = interpersonal manipulation factor.

egocentricity and affective responsiveness. This class is labeled the "high cognitive and moderate other factors psychopathy group".

Figure 3 shows the profile plot for the four-class solution (means and standard deviations are presented in Table 3) for university student sample. Class 1 (63.4\% of students) is the largest group. It is characterized by low mean scores on affective responsiveness, cognitive responsiveness, egocentricity, and moderate scores on interpersonal manipulation, and is labeled the "low psychopathy group". Class $2(13.7 \%)$ is characterized by low mean scores on affective responsiveness and 


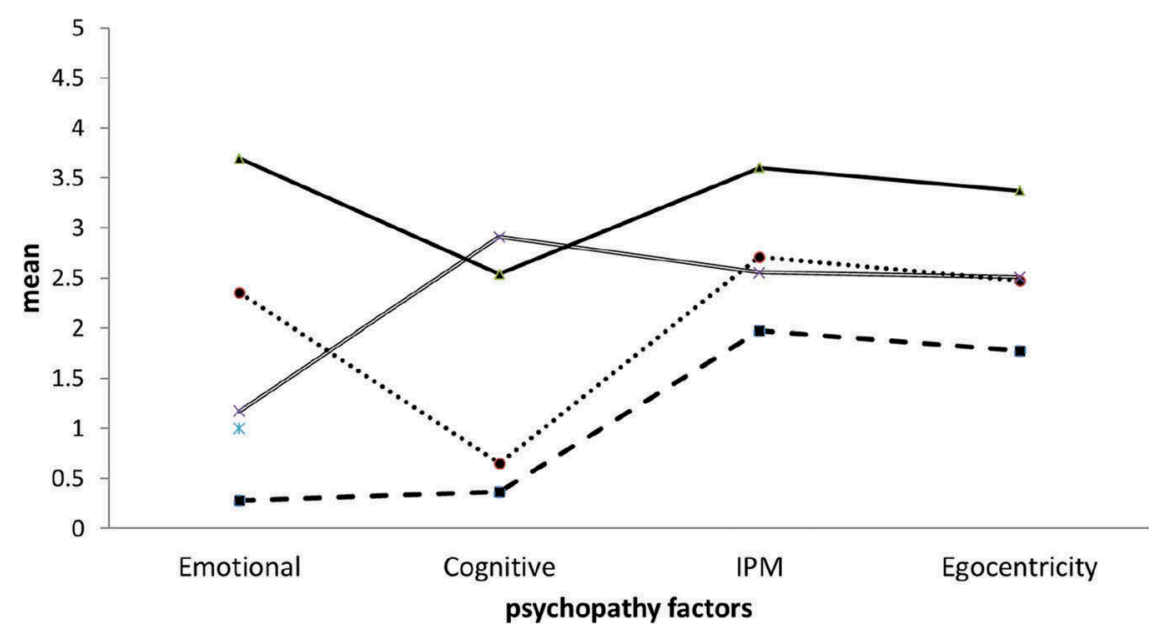

Figure 3. Latent profile analysis plot of psychopathic traits in university sample $(N=2080)$. Dashed line - Class $1=$ "low psychopathy group" (63.4\% of cases); Double solid line - Class 2 = "low affective responsiveness, and moderate other factor psychopathy group" (13.7\%); Dotted line - Class 3 = "low cognitive responsiveness, and moderate other factors psychopathy group" (15.5\%); Solid line - Class $4=$ "high psychopathy group" (7.4\%); Affective = affective responsiveness factor; Cognitive $=$ cognitive responsiveness factor; IPM $=$ interpersonal manipulation factor.

moderate scores on cognitive responsiveness, interpersonal manipulation and egocentricity. This class is labeled the "low affective responsiveness, and moderate other factor psychopathy group". Class 3 (15.5\% of students) is characterized by low mean scores on cognitive responsiveness, and moderate affective responsiveness, interpersonal manipulation and egocentricity. This class is labeled the "low cognitive responsiveness, and moderate other factors psychopathy group". Class 4 (7.4\%) is the smallest group. It is characterized by high mean scores on affective responsiveness, interpersonal manipulation, egocentricity and moderate scores on cognitive responsiveness. This class is labeled the "high psychopathy group".

Figure 4 shows the profile plot for the 4-class solution (means and standard deviations are presented in Table 3) for the sample of adolescents. Class 1 (4.6\% of young people) is characterized by low mean scores on psychopathy factors. This class is labeled the "low psychopathy group". Class $2(35.1 \%)$ is characterized by moderate scores on all psychopathy factors. This class is labeled the "moderate psychopathy group". Class $3(47.8 \%)$ is characterized by low scores on interpersonal manipulation and egocentricity, and moderate scores on affective responsiveness and high on cognitive responsiveness. This class is labeled the "high cognitive responsiveness psychopathy group". Class $4(12.4 \%)$ is characterized by high mean scores on interpersonal manipulation and moderate on affective/cognitive responsiveness and egocentricity. This class is labeled the "moderate psychopathy with high interpersonal manipulation traits group".

\section{Discussion}

The aim of the current study was to compare prisoners, community adults, university students, and adolescents on four psychopathy dimensions (affective responsiveness, cognitive responsiveness, interpersonal manipulation, and egocentricity). Another aim was to identify meaningful psychopathic trait constellations among the four samples of participants, using latent profile analysis (LPA). In light of recent evidence that antisocial/criminal behavior may be an outcome rather than a fundamental part of psychopathy (e.g., Boduszek and Debowska 2016;Boduszek, Debowska, and Willmott 2017; Cooke and Michie 2001; Skeem and Cooke 2010a, 2010b), we used a personalityderived psychopathy measure, the Psychopathic Personality Traits Scale (PPTS; Boduszek et al. 


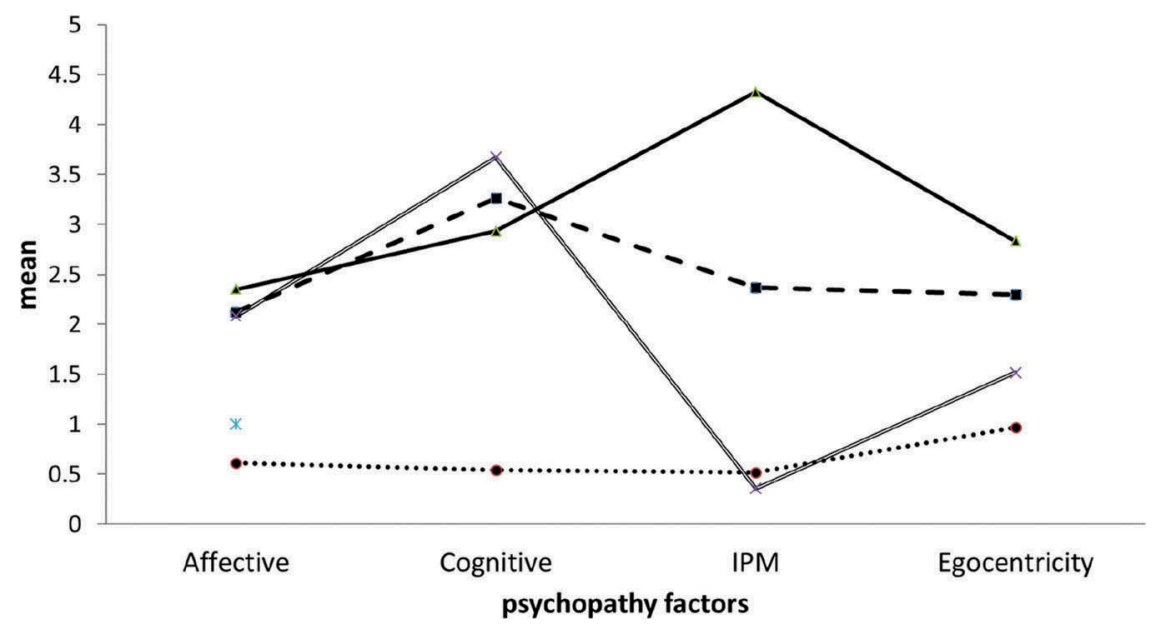

Figure 4. Latent profile analysis plot of psychopathic traits in adolescent sample $(N=472)$. Dotted line - Class $1=$ "low psychopathy group" (4.6\% of cases); Dashed line - Class 2 = "moderate psychopathy group" (35.1\%); Double solid line - Class $3=$ "high cognitive responsiveness psychopathy group" (47.8\%); solid line - Class $4=$ "moderate psychopathy with high interpersonal manipulation traits group" (12.4\%); Affective = affective responsiveness factor; Cognitive = cognitive responsiveness factor; IPM = interpersonal manipulation factor.

2016a), for all analyses. ANOVA results revealed some significant differences in trait intensity across samples. LPA demonstrated that permutations of psychopathic personality traits are not identical across populations. However, all groups had a low and high psychopathy class. The number of participants classed as highly psychopathic was comparable across samples.

Analyses demonstrated significant group differences on all psychopathy variables. Adolescents evidenced greater deficits in affective and cognitive responsiveness than the remaining groups of participants, which may suggest that the two sets of traits develop into adulthood. Affective and cognitive responsiveness are conceptually related to affective and cognitive empathy constructs and prior research showed age-related differences in brain activation when participants are exposed to empathy-eliciting stimuli. Specifically, affective processing shifts from limbic areas in childhood to the involvement of prefrontal cortex in adulthood - a process related to the later maturation of prefrontal systems (Decety and Michalska 2010). It therefore appears that affective and cognitive responsiveness may rely on brain regions which are not yet fully functional in adolescence. Although this assertion needs to be investigated using fMRI data, some of the core psychopathy traits may be malleable. This offers a promising avenue for interventions aimed at young people identified as at risk of adult psychopathy. Another interesting finding pertains to higher scores on interpersonal manipulation psychopathy dimension among university students, compared with adolescents and prisoners. Although speculative at this stage, this may be due to higher cognitive abilities (e.g., intelligence) expected of university students. In keeping with this explanation is a study by Boduszek, Debowska, and Willmott (2017), which demonstrated that prisoners classed in "high interpersonal manipulation" group are likely to be convicted of white collar crimes, i.e., crimes associated with higher social class background.

The results of LPA yielded a 4-class solution for the full sample of participants and all subsamples. The inspection of qualitative properties of the identified classes, however, revealed some differences across the four populations. Alike high psychopathy groups were identified for prisoners and university students. With $7.6 \%$ and $7.4 \%$ of participants respectively, these groups were nearly identical in size, suggesting that increased psychopathy is not more common among forensic than non-forensic populations. Although not identical, a comparable group has also been identified among adolescents. Specifically, $12.4 \%$ of participants classed in the "moderate psychopathy and high interpersonal manipulation traits" group evidenced similar levels of affective responsiveness 
and egocentricity, smaller deficits in affective responsiveness, and higher egocentricity, compared with highly psychopathic prisoners and students. Adolescents in this group can be seen as those at risk of developing full-blown psychopathic traits in adulthood and hence should be the group targeted for early intervention. Since high psychopathy is identified in approximately $7 \%$ of adult populations, it appears that some of those traits may naturally alleviate in the process of maturation and socialization. Finally, one comparable group was not identified in community adults. Rather, we uncovered two groups which combined the qualities of a high psychopathy group. These were "high interpersonal manipulation and egocentricity psychopathy" group (6.9\% of cases) and "high cognitive responsiveness and moderate other psychopathy factors" group (6.9\%). The two classes differ most prominently on cognitive responsiveness scores, which may be a function of differences in intelligence between members of the groups. Indeed, Boduszek and Debowska (2016) as well Boduszek, Debowska, and Willmott (2017) indicated that psychopathy should be studied along with intelligence because highly intelligent psychopaths may learn to understand others' emotions and respond to others in a socially desirable manner, without increasing their affective engagement. Future research with the PPTS should control for intelligence to verify this claim.

In line with our predictions and Boduszek, Debowska, and Willmott (2017) findings, a low psychopathy group was identified among all samples. This was the highest membership group for prisoners (53.7\%), community adults (73\%), and students (63.4\%). Patterns of scores for prisoners in the low psychopathy group were almost identical to those reported for a corresponding group in Boduszek, Debowska, and Willmott (2017) study with Polish prisoners. Interestingly, low psychopathy group in adolescents was characterized by similarly low scores across PPTS dimensions, but it incorporated merely $4.6 \%$ of all participants. The most numerous group for the sample was the "high cognitive responsiveness psychopathy" group (47.8\% of participants), characterized by low scores on interpersonal manipulation and egocentricity, moderate deficits in affective responsiveness, and high deficits in cognitive responsiveness. This finding confirms our earlier assertion that affective responsiveness and cognitive responsiveness develop later in life, which may be associated with the late maturation of prefrontal cortex.

Although our results for non-forensic samples cannot be evaluated against prior research with similar populations, we can make direct comparisons between Boduszek, Debowska, and Willmott (2017) study and the current LPA results for the prisoner sample. As indicated above, we identified corresponding high and low psychopathy groups to those described in prior research. We also uncovered the "moderate egocentricity and high interpersonal manipulation" (24.9\% of cases) and "moderate psychopathy" (13.8\%) groups whose PPTS scores largely mirrored those reported for Boduszek et al.'s "high interpersonal manipulation" (20.8\%) and "moderate affective/cognitive responsiveness" groups respectively. Only one pattern of scores found for prisoners in prior research, labeled the "moderate psychopathy" group, was not discovered in the present sample of offenders. A similar group, however, was identified in the community sample (labeled the "high interpersonal manipulation and egocentricity psychopathy group"). These findings combined suggest greater stability in the constellations of psychopathic traits across populations at the ends of psychopathy continuum, and greater cross-sample variations in the middle of the continuum.

The present study has several limitations. Firstly, all responses were self-reported, which could have resulted in response bias. However, prior research relying on clinicians' judgment with regards to psychopathy assessment, could also result in skewed findings due to psychopaths' manipulativeness. Secondly, we did not control for participants' IQ, which can be a significant factor in the expression of certain psychopathic traits (see Bate et al. 2014). Finally, future research should assess external criteria which could be correlated with resultant LPA classes, to build a better understanding of risk factors and/or consequences of different psychopathy group membership.

In conclusion, the present findings reveal differences in psychopathic trait intensity across populations, with most pronounced deficits in affective and cognitive responsiveness among adolescents compared with adults. Additionally, the current study provides further evidence for the existence of qualitatively diverse groups of psychopathy. Although certain variations in the patterns 
of scores were detected across samples, we identified a low psychopathy group for all samples, clear high psychopathy groups for prisoners and university students, and increased psychopathy groups among community adults and adolescents. Notably, the membership of the high psychopathy group for prisoner and student samples amounted to approximately 7\%, suggesting that prisoners are not more psychopathic than non-prisoners. This provides important empirical evidence against including criminal/antisocial behavior in psychopathy assessment. Lastly, it was suggested that the most prominent variations in the expression of psychopathic traits can be found in the middle of psychopathy continuum, with more stability across populations at both ends of the continuum.

\section{Conflict of Interest}

Authors declare that they have no conflict of interest.

\section{Notes on contributors}

Daniel Boduszek, $\mathrm{PhD}$ is a professor of criminal psychology at the University of Huddersfield and Director of Quantitative Research Methods Training Unit. His current research interests and publications include the aspects of criminal social identity, homicidal behavior, psychopathy, prisonization, and recidivism.

Agata Debowska, $\mathrm{PhD}$ is a lecturer in psychology at the University of Sheffield. Her current research interests and publications include violence against women and children, child sexual abuse, psychopathy, and criminal social identity.

Nicole Sherretts, PhD is a data analyst in Office of the State's Attorney for Baltimore City. Her research interest an publications include criminal social identity, recidivism, and psychopathy.

Dominic Willmott, $\mathrm{PhD}$ is a research fellow at the University of Huddersfield (None in Three Research Center). His current research interests and publications include gender biased violence and jury decision making.

Mike Boulton, $\mathrm{PhD}$ is an emeritus professor of psychology at the University of Chester. His current research interests and publications include children's social relationships and their links with adjustment.

Krzysztof Kielkiewicz, $\mathrm{PhD}$ is a lecturer in psychology at the University of Finance and Management. His current research interests and publications include psychopathy and spirituality.

Katarzyna Popiolek, PhD is an associate professor and dean of psychology school at the SWPS University of Social Sciences and Humanities. Her current research interests and publications include interpersonal relationships.

Philip Hyland, $\mathrm{PhD}$ is a senior lecturer in psychology at the Maynooth University. His current research interests and publications include the impact of traumatic life events on people's lives.

\section{ORCID}

Daniel Boduszek (1) http://orcid.org/0000-0001-5863-2906

\section{References}

Akaike, Hirotugu. 1987. "Factor Analysis and AIC." Selected Papers of Hirotugu Akaike 52 (3):317-32. doi:10.1007/ BF02294359.

American Psychiatric Association. 2013. Diagnostic and Statistical Manual of Mental Disorders (DSM-5 $)$. Washington, DC: American Psychiatric Pub.

Bate, Carolyn, Daniel Boduszek, Katie Dhingra, and Christopher Bale. 2014. "Psychopathy, Intelligence and Emotional Responding in a Non-forensic Sample: An Experimental Investigation." The Journal of Forensic Psychiatry and Psychology 25 (5):600-12. doi:10.1080/14789949.2014.943798.

Boduszek, Daniel and Agata Debowska. 2016. "Critical Evaluation of Psychopathy Measurement (PCL-R and SRP-III/ SF) and Recommendations for Future Research.” Journal of Criminal Justice 44:1-12. doi:10.1016/j. jcrimjus.2015.11.004. 
Boduszek, Daniel, Agata Debowska, and Dominic Willmott. 2017. "Latent Profile Analysis of Psychopathic Traits among Homicide, General Violent, Property, and White-collar Offenders.” Journal of Criminal Justice 51:17-23. doi:10.1016/j.jcrimjus.2017.06.001.

Boduszek, Daniel, Agata Debowska, and Dominic Willmott. 2018. "Psychopathic Personality Traits Model (PPTM): A New Approach to Defining Psychopathy.” Pp. 216-24 in Matt DeLisi (Ed.), Routledge International Handbook of Psychopathy and Crime. New York, NY: Routledge Taylor \& Francis Group.

Boduszek, Daniel, Agata Debowska, Katie Dhingra, and Matt DeLisi. 2016a. "Introduction and Validation of Psychopathic Personality Traits Scale (PPTS) in a Large Prison Sample." Journal of Criminal Justice 46:9-17. doi:10.1016/j.jcrimjus.2016.02.004.

Boduszek, Daniel, Agata Debowska, Nicole Sherretts, and Dominic Willmott. 2018. "Psychopathic Personality Traits Scale (PPTS): Construct Validity of the Instrument in a Sample of US Prisoners." Frontiers in Psychology 9. doi:10.3389/fpsyg.2018.01596.

Boduszek, Daniel, Katie Dhingra, Philip Hyland, and Agata Debowska. 2016b. "A Bifactorial Solution to the Psychopathy Checklist: Screening Version in A Sample of Civil Psychiatric Patients." Criminal Behaviour and Mental Health 26 (3):174-85. doi:10.1002/cbm.1956.

Buzina, Nadica. 2012. "Psychopathy-Historical Controversies and New Diagnostic Approach." Psychiatria Danubina 24 (2):134-42. (https://hrcak.srce.hr/106215).

Cohen, Jacob. 1988. Statistical Power Analysis for the Social Sciences. Hillsdale, N.J.: Lawrence Erlbaum Associates.

Cooke, David J. and Christine Michie. 2001. "Refining the Construct of Psychopathy: Towards a Hierarchical Model." Psychological Assessment 13 (2):171. doi:10.1037/1040-3590.13.2.171.

De Fruyt, Filip and Barbara De Clercq. 2014. "Antecedents of Personality Disorder in Childhood and Adolescence: Toward an Integrative Developmental Model.” Annual Review of Clinical Psychology 10:449-76. doi:10.1146/ annurev-clinpsy-032813-153634.

Debowska, Agata, Daniel Boduszek, Katie Dhingra, Nicole Sherretts, Dominic Willmott, and Matt DeLisi. 2018. "Can We Use Hare's Psychopathy Model within Forensic and Non-forensic Populations? an Empirical Investigation." Deviant Behavior 39 (2):224-42. doi:10.1080/01639625.2016.1266887.

Decety, Jean and Kalina J. Michalska. 2010. "Neurodevelopmental Changes in the Circuits Underlying Empathy and Sympathy from Childhood to Adulthood." Developmental Science 13 (6):886-99. doi:10.1111/j.14677687.2009.00940.x.

Edens, John F., Jennifer L. Skeem, Keith R. Cruise, and Elizabeth Cauffman. 2001. "Assessment of "juvenile Psychopathy" and Its Association with Violence: A Critical Review.” Behavioral Sciences \& the Law 19 (1):53-80. doi:10.1002/bsl.425.

Frick, Paul J. 2007. "Using the Construct of Psychopathy to Understand Antisocial and Violent Youth.” Pp. 343-67 in Hugues Herve \& John C. Yuille (Eds.), The Psychopath: Theory, Research, and Practice. New York, NY: Routledge

Hare, Robert D. 1980. "A Research Scale for the Assessment of Psychopathy in Criminal Populations." Personality and Individual Differences 1 (2):111-19. doi:10.1016/0191-8869(80)90028-8.

Hare, Robert D. 1991. The Hare Psychopathy Checklist-revised: Manual. Toronto: Multi-Health Systems, Incorporated.

Hare, Robert D. 2003. The Hare Psychopathy Checklist-revised. 2: Manual. Toronto: Multi-Health Systems, Incorporated.

Hare, Robert D. and Craig S. Neumann. 2008. "Psychopathy as a clinical and empirical construct." Annual Review of Clinical Psychology 4:217-246. doi:10.1146/annurev.clinpsy.3.022806.091452.

Lilienfeld, Scott O. and Hal Arkowitz. 2007. "What "Psychopath" Means." Scientific American Mind 18 (6):80-81. doi:10.1038/scientificamericanmind1207-80.

Lo, Yungtai, Nancy R. Mendell, and Donald B. Rubin. 2001. “Testing the Number of Components in a Normal Mixture." Biometrika 88 (3):767-78. doi:10.1093/biomet/88.3.767.

Marcus, Robert F. 2017. The Development of Aggression and Violence in Adolescence. New York, NY: Palgrave Macmillan.

Nylund, Karen L., Tihomir Asparouhov, and Bengt O. Muthén. 2007. "Deciding on the Number of Classes in Latent Class Analysis and Growth Mixture Modeling: A Monte Carlo Simulation Study." Structural Equation Modeling: A Multidisciplinary Journal 14 (4):535-69. doi:10.1080/10705510701575396.

Ogloff, James RP. 2006. "Psychopathy/antisocial Personality Disorder Conundrum." Australian and New Zealand Journal of Psychiatry 40 (6-7):519-28. doi:10.1080/j.1440-1614.2006.01834.x.

Patrick, Christopher J. 2007. "Back to the Future: Cleckley as a Guide to the Next Generation of Psychopathy Research.” Pp. 605-17 in Handbook of Psychopathy, edited by C. Patrick. New York: Guilford Press,

Patrick, Christopher J and Laura E. Drislane. 2015. “Antisocial Personality Disorder and Psychopathy.” Pp. 681-706 in Oxford Textbook of Psychopathology, edited by P. H. Blaney, R. F. Krueger, and T. Millon. New York, NY: Oxford University Press,

Patrick, Christopher J., Brian M. Hicks, Penny E. Nichol, and Robert F. Krueger. 2007. “A Bifactor Approach to Modeling the Structure of the Psychopathy Checklist-revised." Journal of Personality Disorders 21 (2):118-41. doi:10.1521/pedi.2007.21.2.118. 
Ramaswamy, Venkatram, Wayne S. DeSarbo, David J. Reibstein, and William T. Robinson. 1993. “An Empirical Pooling Approach for Estimating Marketing Mix Elasticities with PIMS Data.” Marketing Science 12 (1):103-24. doi:10.1287/mksc.12.1.103.

Rogers, Richard. 1995. Diagnostic and Structured Interviewing: A Handbook for Psychologists. Odessa, FL: Psychological Assessment Resources.

Schwarz, Gideon. 1978. "Estimating the Dimension of a Model." The Annals of Statistics 6 (2):461-64. (http:// projecteuclid.org/euclid.aos/1176344136).

Sclove, Stanley L. 1987. "Application of Model-selection Criteria to Some Problems in Multivariate Analysis." Psychometrika 52 (3):333-43. doi:10.1007/BF02294360.

Sharratt, Kathryn, Daniel Boduszek, and Christopher Retzler. 2019. "Clarifying the Relationship between Psychopathy and Intelligence Using Four Dimensions of the WASI-II." Deviant Behavior 1-9. doi:10.1080/ 01639625.2019 .1582968 .

Shevlin, Mark and Ask Elklit. 2008. “A Latent Class Analysis of Adolescent Adverse Life Events Based on A Danish National Youth Probability Sample.” Nordic Journal of Psychiatry 62 (3):218-24. doi:10.1080/08039480801983992.

Skeem, Jennifer L. and David J. Cooke. 2010a. "Is Criminal Behavior a Central Component of Psychopathy? Conceptual Directions for Resolving the Debate." Psychological Assessment 22 (2):433-45. doi:10.1037/a0008512.

Skeem, Jennifer L. and David J. Cooke. 2010b. "One Measure Does Not a Construct Make: Directions toward Reinvigorating Psychopathy Research-Reply to Hare and Neumann (2010)." Psychological Assessment 22 (2):455-59. doi:10.1037/a0014862.

Woodworth, Michael and Stephen Porter. 2002. "In Cold Blood: Characteristics of Criminal Homicides as a Function of Psychopathy." Journal of Abnormal Psychology 111 (3):436-45. doi:10.1037/0021-843X.111.3.436.

Yuan, Ke-Hai and Peter M. Bentler. 2000. "Three Likelihood-based Methods for Mean and Covariance Structure Analysis with Nonnormal Missing Data.” Sociological Methodology 30 (1):165-200. doi:10.1111/0081-1750.00078. 